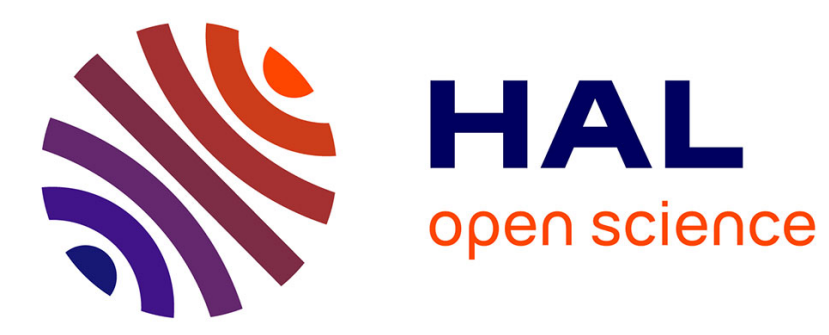

\title{
Réalisation et modélisation Monte-Carlo du Transistor Bipolaire à Hétérojonctions NnpnN InGaAlAs/InGaAs/InGaAlAs
}

\author{
J.L. Pelouard, P. Hesto, J.P. Praseuth, L. Goldstein
}

\section{To cite this version:}

J.L. Pelouard, P. Hesto, J.P. Praseuth, L. Goldstein. Réalisation et modélisation Monte-Carlo du Transistor Bipolaire à Hétérojonctions NnpnN InGaAlAs/InGaAs/InGaAlAs. Revue de Physique Appliquée, 1987, 22 (8), pp.905-911. 10.1051/rphysap:01987002208090500 . jpa-00245631

HAL Id: jpa-00245631

https://hal.science/jpa-00245631

Submitted on 1 Jan 1987

HAL is a multi-disciplinary open access archive for the deposit and dissemination of scientific research documents, whether they are published or not. The documents may come from teaching and research institutions in France or abroad, or from public or private research centers.
L'archive ouverte pluridisciplinaire HAL, est destinée au dépôt et à la diffusion de documents scientifiques de niveau recherche, publiés ou non, émanant des établissements d'enseignement et de recherche français ou étrangers, des laboratoires publics ou privés. 


\title{
Réalisation et modélisation Monte-Carlo du Transistor Bipolaire à Hétérojonctions NnpnN InGaAlAs/InGaAs/InGaAlAs
}

\author{
J. L. Pelouard $\left({ }^{+}\right)$, P. Hesto $\left({ }^{+}\right)$, J. P. Praseuth $\left({ }^{\circ}\right)$ and L. Goldstein $\left({ }^{\circ},{ }^{*}\right)$ \\ $\left(^{+}\right)$Laboratoire de Microstructures et de Microélectronique (CNRS) \\ $\left({ }^{\circ}\right)$ Laboratoire de Bagneux (CNET) \\ Groupement Scientifique CNET/CNRS, 196 avenue H. Ravera, 92220 Bagneux, France
}

(Reçu le 16 janvier 1987, accepté le 24 février 1987)

\begin{abstract}
Résumé. - Ce papier présente une étude expérimentale et une analyse par simulation Monte-Carlo de Transistors Bipolaires à Hétérojonctions InGaAlAs/InGaAs/InGaAlAs avec et sans espaceurs. Ces espaceurs, destinés à séparer les jonctions électriques et métallurgiques, permettent de diminuer les hauteurs de barrière vues par les électrons tout en conservant celles vues par les trous. L'injection, de nature thermoïonique pour les hétérojonctions abruptes, se fait par effet diffusif lorsque l'on introduit un espaceur, et ainsi le coefficient d'injection est augmenté. D'autre part les espaceurs au collecteur conduisent à une meilleure collection des électrons, cette collection étant facilitée par une injection d'électrons chauds dans le cas d'une hétérojonction émetteur-base abrupte. Les gains en courant en émetteur commun obtenus sont supérieurs à 100 .
\end{abstract}

\begin{abstract}
An experimental study of Heterojonction Bipolar Transistor InGaAlAs/InGaAs/InGaAlAs with and without spacers will be reported and the results will be interpreted using Monte-Carlo simulations. By a separation between the metallurgical junction and the electrical one, these spacers reduce the barrier height for the electrons but preserve the barrier height for the holes. The injection is a thermionic one for the abrupt heterojonctions and a diffusive one for the heterojunctions with a spacer and then the injection coefficient is higher. Moreover the collection is higher for the spacer collector-base junctions, especially when the electrons are the hot electrons injected from an abrupt emitter junction. The common emitter configuration current gains are greater than 100 .
\end{abstract}

\section{Introduction.}

Une hétérojonction idéale entre un semi-conducteur à grande bande interdite de type $\mathrm{N}$ et un semiconducteur à faible bande interdite de type $\mathrm{P}$ permet théoriquement d'obtenir une efficacité d'injection pour les électrons très proche de l'unité même avec une zone $\mathrm{P}$ comportant une forte densité d'atomes accepteurs [1]. En effet la barrière de potentiel vue par les trous est très supérieure à celle vue par les électrons en raison de la différence des largeurs des bandes interdites (Fig. 1). Un transistor bipolaire à hétérojonction dont la jonction Emetteur-Base est constituée d'une hétérojonction semi-conducteur à grande bande interdite/semi-conducteur à faible bande interdite doit donc permettre d'obtenir des gains en courant importants même en surdopant la

(*) Actuellement à la CGE Marcoussis.

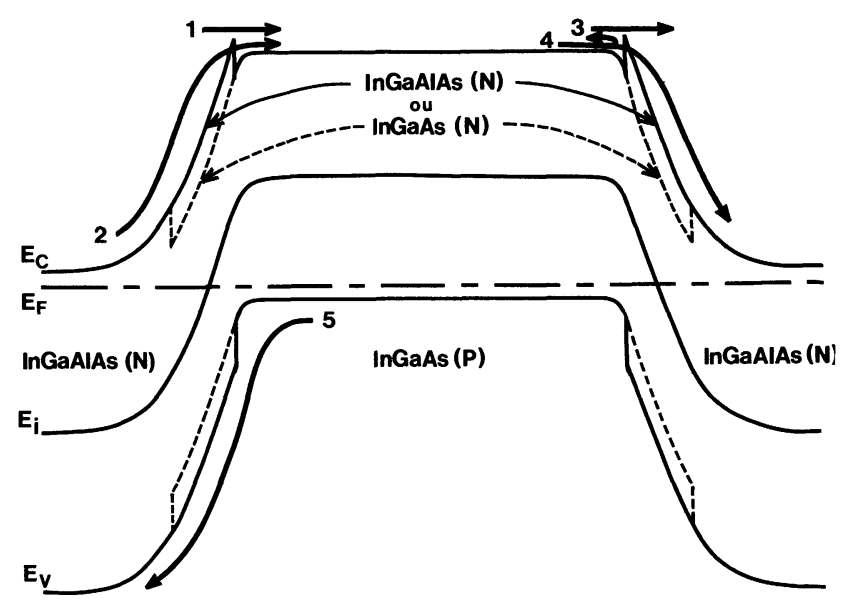

Fig. 1. - Diagramme des bandes du transistor non polarisé.

[Band diagram for the transistors without bias voltages.] 
base, c'est-à-dire avec des résistances d'accès suffisamment faibles pour atteindre des fréquences de coupure élevées. D'autre part la symétrie des Transistors Bipolaires à Double Hétérojonctions est intéressante pour des circuits logiques $\mathrm{I}^{2} \mathrm{~L}$ et ECL. Néanmoins les performances des transistors sont souvent masquées par les phénomènes de recombinaison aux hétérojonctions, ce qui fait chuter le coefficient d'injection, et par la mauvaise collection des électrons en raison de la présence d'une barrière de potentiel qui s'oppose à leur passage à la jonction Base-Collecteur (spike, Fig. 1, courant 4). Une solution permet de s'affranchir de ces limitations : elle consiste à diminuer les barrières de potentiel vues par les électrons, ce qui conduit à augmenter le courant participant à l'effet transistor par rapport au courant de recombinaison et à améliorer la collection. Technologiquement cette diminution peut être obtenue, soit par la réalisation d'hétérojonctions graduelles en composition [2, 3], soit par l'introduction d'une mince couche de semi-conducteur à faible bande interdite aux hétérojonctions (espaceur) [4]. C'est cette dernière solution que nous avons choisie en raison de la difficulté à réaliser des hétérojonctions graduelles en Epitaxie par Jets Moléculaires avec des semi-conducteurs en accord de maille avec InP.

Pour étudier l'influence de ces espaceurs sur les caractéristiques des transistors, nous avons réalisé des Transistors Bipolaires à Hétérojonctions InGaAlAs/InGaAs/InGaAlAs sans espaceur (TBDH), avec un espaceur au collecteur (TBHS) et avec deux espaceurs à l'émetteur et au collecteur (TBHDS) et nous avons interprété les caractéristiques obtenues à l'aide de simulations particulaires de type Monte-Carlo.

\section{Réalisation et étude théorique.}

2.1 TECHNOLOGIE ET GÉOMÉTRIE DES TRANSISTORS. - Une coupe schématique des transistors réalisés est représentée sur la figure 2 . Les couches sont épitaxiées par jets moléculaires. Le matériau à faible bande interdite (la base) est $\mathrm{In}_{0,53} \mathrm{Ga}_{0,47} \mathrm{As}$ en

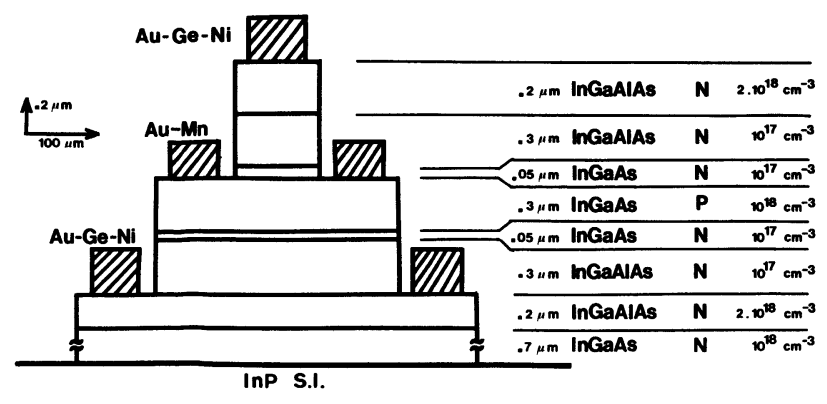

Fig. 2. - Coupe schématique des transistors.

[Schematic cross section for the transistors.] raison de ses qualités très prometteuses pour l'électronique rapide (masse effective des électrons faible, $m=0,041 m_{0}$, et grande distance en énergie entre la vallée $\Gamma$ et les vallées latérales $L, \Delta E_{\Gamma L}=0,5 \mathrm{eV}$ ) et pour l'optoélectronique (la longueur d'onde associée à la largeur de la bande interdite correspond au minimum d'absorption des fibres optiques). Le semi-conducteur à grande bande interdite est $\mathrm{In}_{0,53} \mathrm{Ga}_{0,28} \mathrm{Al}_{0,19} \mathrm{As}$. Ce composé quaternaire a été préféré à $\mathrm{In}_{0,52} \mathrm{Al}_{0,48} \mathrm{As}$ en raison de ses meilleures qualités cristallines et électriques dues à sa plus faible teneur en aluminium [5]. Les structures, isolées en technologie MESA, ont été obtenues à l'aide de techniques comparables à celles utilisées pour les composants à base de GaAs et GaAlAs [6].

2.2 LA Simulation Monte-Carlo. - Le principe de la simulation est le suivant [7].Connaissant les valeurs des probabilités des différentes interactions des porteurs avec les imperfections du réseau cristallin (phonons, impuretés, alliage) un tirage au sort permet de déterminer le temps de vol libre des porteurs. Il est alors possible de les faire se déplacer sous l'effet du champ électrique auquel ils sont soumis en appliquant les lois de la dynamique. A la fin de leur vol libre, ils subissent une interaction dont la nature est tirée au sort, et on est alors en mesure de déterminer leur nouvelle vitesse et leur nouvelle énergie. Régulièrement on calcule la nouvelle répartition du champ électrique due aux porteurs et aux polarisations appliquées. Cette méthode de simulation, qui permet de suivre au cours du temps, la position, la vitesse et l'énergie des porteurs, rend parfaitement compte de l'histoire des porteurs au cours de leur mouvement et prend naturellement en compte les phénomènes de transport non-stationnaires.

\section{Phénomènes d'injection aux hétérojonctions.}

3.1 CARACTÉRISTIQUES EXPÉRIMENTALES. - Un exemple de caractéristiques $I(V)$ des hétérojonctions réalisées polarisées en direct est représenté sur la figure 3. Cet exemple est relatif aux deux jonctions $\mathrm{du}$ transistor à un espaceur. La jonction émetteurbase est donc une hétérojonction abrupte et la jonction base-collecteur une hétérojonction avec espaceur. On remarque que le courant de la jonction collecteur-base est de 100 fois supérieur à celui de la jonction émetteur-base (le rapport des surfaces n'est que de 8) et qu'il y a une saturation des courants à fort niveau due à une résistance série. Les caractéristiques présentent deux parties linéaires, avec un coefficient d'idéalité plus important à faible niveau, ce phénomène étant plus marqué pour la caractéristique collecteur-base.

3.2 RÉsultats DES Simulations MonteCARLO. - Pour caractériser les phénomènes de 


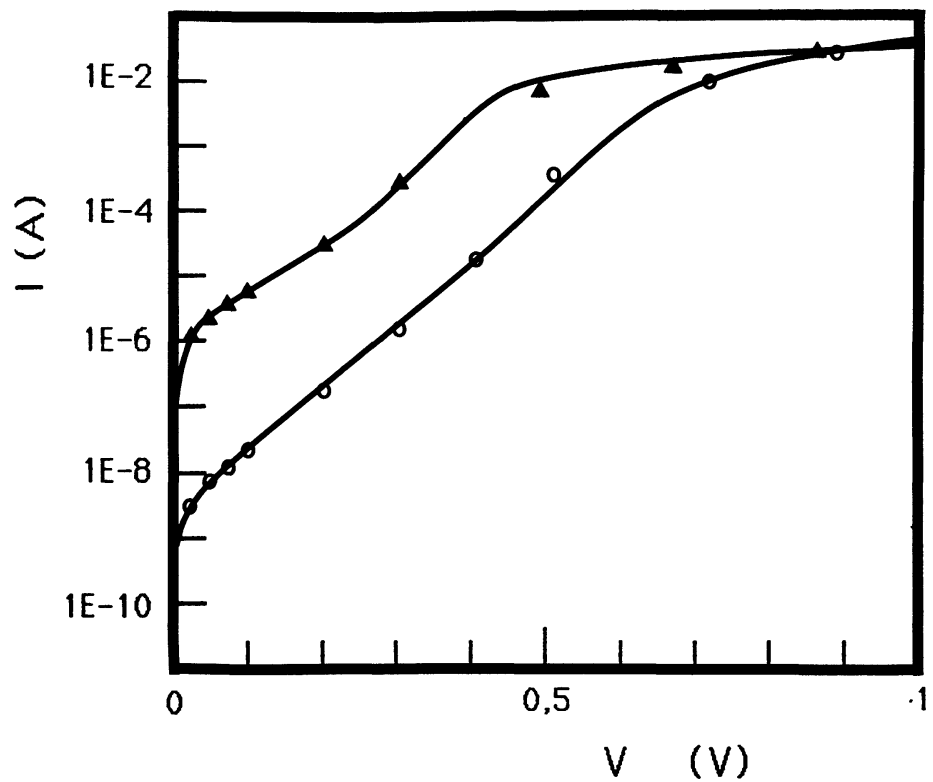

Fig. 3. - Caractéristiques $I(V)$ des jonctions du transistor à un espaceur. Courbes: relevés expérimentaux. Points : valeurs calculées à l'aide de l'expression (9) avec les paramètres donnés dans le tableau $\mathrm{I}$; $\mathrm{O}$ : hétérojonction émetteur-base (sans espaceur, surface égale à $1,44 \times$ $10^{-4} \mathrm{~cm}^{2}$ ), $\Delta$ : hétérojonction base-collecteur (avec espaceur, surface égale à $1,16 \times 10^{-3} \mathrm{~cm}^{2}$ ).

$[I(V)$ characteristics for the junctions of the one spacer transistor. Curves are the experimental values. Points are from equation (9) with parameters given in table $\mathrm{I}$; $\mathrm{O}$ : emitter-base heterojunction (without spacer, area equal to $1.44 \times 10^{-4} \mathrm{~cm}^{2}$ ), $\Delta:$ base-collector heterojunction (with spacer, area equal to $\left.1.16 \times 10^{-3} \mathrm{~cm}^{2}\right)$.]

conduction dans ces deux hétérojonctions nous avons simulé séparément leur fonctionnement en polarisation directe, et nous avons relevé la distribution en vitesse des électrons dans la base à $0,1 \mu \mathrm{m}$ de la jonction électrique. Les spectres correspondants sont représentés sur la figure 4. Le spectre relatif à la jonction avec espaceur (courbe 1) est symétrique et centré autour d'une faible vitesse $(v=4 \times$ $\left.10^{6} \mathrm{~cm} / \mathrm{s}\right)$. La température de la population est proche de celle du réseau. Nous pouvons conclure à une population d'électrons en quasi-équilibre et à une injection depuis le semi-conducteur à grande bande interdite (l'émetteur) par effet diffusif. Ce résultat est en accord avec la forme du diagramme des bandes (Fig. 1, courant 2) et montre que l'hétérojonction n'agit que très peu sur l'injection. La fome du spectre relatif à des électrons injectés à travers une hétérojonction abrupte sans espaceur est très différente (courbe 2). Ce spectre comporte deux pics. L'un, étroit et bien défini, est centré autour d'une vitesse élevée $\left(v=7,5 \times 10^{7} \mathrm{~cm} / \mathrm{s}\right.$, soit une énergie cinétique de $65 \mathrm{meV}$ ). Il correspond aux électrons n'ayant subi aucune interaction et qui ont une énergie cinétique proche de l'énergie potentielle qu'ils ont gagné depuis leur franchissement de

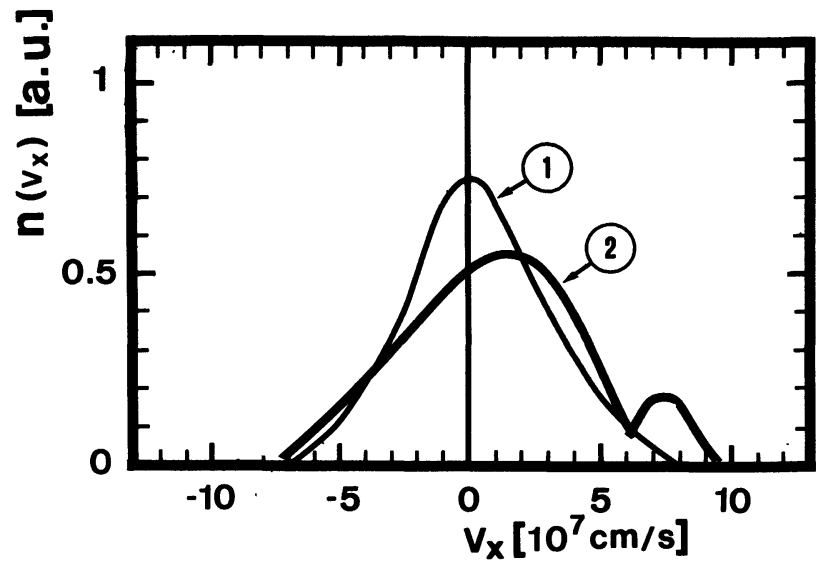

Fig. 4. - Distribution des électrons de la base injectés depuis l'émetteur en fonction de leur vitesse suivant la direction du champ électrique. Courbe 1 : jonction avec espaceur, courbe 2 : jonction sans espaceur. Les spectres sont relevés à $0,1 \mu \mathrm{m}$ de l'émetteur.

[The electron distribution in the base at $0.1 \mu \mathrm{m}$ from the emitter as a function of the electron velocities along the electric field. Curve 1 : junction with a spacer, curve 2 : junction without spacer.]

l'hétérojonction. L'autre, pratiquement symétrique et centré autour d'une faible vitesse $(v=5 \times$ $10^{6} \mathrm{~cm} / \mathrm{s}$ ), correspond aux électrons qui ont subi des interactions qui redistribuent de façon isotrope leurs vecteurs vitesses (impuretés et alliage). On peut remarquer qu'il n'y a pratiquement aucun électron ayant une vitesse négative suffisante pour rétrodiffuser vers le semi-conducteur à grande bande interdite. Ce résultat est en accord avec la forme du diagramme des bandes (Fig. 1, courant 1 ) et correspond à une injection de type thermoionique comparable à ce qui se passe dans une jonction Métal-Semi-conducteur, le semi-conducteur à faible bande interdite jouant ici le rôle du métal et celui à grande bande interdite celui du semi-conducteur [8].

3.3 COURANTS À TRAVERS LES HÉtÉROJONCTIONS. - Nous allons d'abord donner les expressions des courants dans les différents cas.

3.3.1 Hétérojonction abrupte. - Nous avons vu que dans ce cas le courant était de nature thermoionique, ce qui donne une densité de courant de la forme :

$$
J=J_{\mathrm{S}}\left(\exp \left(V / n k_{\mathrm{B}} T\right)-1\right)
$$

avec

$$
J_{\mathrm{S}}=q N_{\mathrm{D}}\left(k_{\mathrm{B}} T / 2 \pi m^{*}\right)^{1 / 2} \exp \left(-\Phi_{0} / n k_{\mathrm{B}} T\right)
$$

où $N_{\mathrm{D}}$ est le dopage et $m^{*}$ la masse effective des électrons dans le semi-conducteur à grande bande interdite, et $\Phi_{0}$ la hauteur de barrière au repos qui est donnée par le modèle d'Anderson :

$\Phi_{0}=\Delta E_{\mathrm{C}}+E_{\mathrm{G} 2}+k_{\mathrm{B}} T \operatorname{Ln}\left(N_{\mathrm{A}} N_{\mathrm{D}} / N_{\mathrm{C} 1} N_{\mathrm{V} 2}\right)$ 
$\Delta E_{\mathrm{C}}$ est la hauteur de la discontinuité de la bande de conduction, $N_{\mathrm{C} 1}$ la densité d'état de la bande de conduction du semi-conducteur à grande bande interdite. $N_{\mathrm{V} 2}$ est la densité d'état de la bande de valence, $N_{\mathrm{A}}$ le dopage et $E_{\mathrm{G} 2}$ la largeur de la bande interdite du semi-conducteur à faible bande interdite.

$n$ est un coefficient prenant en compte la répartition du potentiel dans les deux semi-conducteurs :

$$
n=1+\varepsilon_{1} N_{\mathrm{D}} / \varepsilon_{2} N_{\mathrm{A}}
$$

avec $\varepsilon_{1}$ et $\varepsilon_{2}$ permittivités des deux semi-conducteurs.

3.3.2 Hétérojonction avec espaceur. - Le courant étant de nature diffusive, la densité de courant est donnée par l'expression de Shockley relative à une diode à base courte (voir les valeurs des longueurs de diffusion des électrons dans la base données dans le tableau I) :

$$
J=J_{\mathrm{S}}\left(\exp \left(V / k_{\mathrm{B}} T\right)-1\right)
$$

avec

$$
J_{\mathrm{S}}=q D_{n} n_{p 0} / W_{\mathrm{B}}
$$

où $D_{n}$ est le coefficient de diffusion des électrons, $n_{p 0}$ la densité d'électrons à l'équilibre et $W_{\mathrm{B}}$ l'épaisseur du semi-conducteur à faible bande interdite.

3.3.3 Courant de recombinaison et de trous. - Nous supposerons un courant de recombinaison de la forme :

$$
J=J_{\mathrm{R}}\left(\exp \left(V / 2 k_{\mathrm{B}} T\right)-1\right)
$$

et un courant de trou (Fig. 1, courant 5) injecté dans les zones $N$ donné par:

$$
\begin{aligned}
J=q D_{p} p_{n 0} \exp \left(-\Delta E_{\mathrm{C}} / k_{\mathrm{B}} T\right) / W_{\mathrm{E}} \times \\
\times\left(\exp \left(V / k_{\mathrm{B}} T\right)-1\right) .
\end{aligned}
$$

Nous verrons que ce courant est toujours négligeable.

3.3.4 Courant total. - Aux effets définis précédemment nous ajouterons la résistance série $R_{\mathrm{S}}$ et une résistance de fuite en parallèle $R_{\mathrm{P}}$. La densité de courant sera alors de la forme :

\begin{tabular}{|c|c|c|c|c|}
\hline & & $\begin{array}{c}\text { TBDH } \\
\text { (0 espaceur) }\end{array}$ & $\begin{array}{c}\text { TBHS } \\
(1 \text { espaceur CB) }\end{array}$ & $\begin{array}{c}\text { TBHDS } \\
\text { (2 espaceurs) }\end{array}$ \\
\hline \multirow{5}{*}{ Emetteur-Base } & $J,\left(\mathrm{~A} / \mathrm{cm}^{2}\right)$ & 9. E-8 & $5 . \mathrm{E}-8$ & 1,6.E-6 \\
\hline & $J_{\mathrm{r}}\left(\mathrm{A} / \mathrm{cm}^{2}\right)$ & 3.E-5 & 2. E-5 & 1,6.E-4 \\
\hline & $P,(\Omega)$ & 35 & 10 & 10 \\
\hline & $R_{\mathrm{p}}(\Omega)$ & 3.E6 & 3.E7 & 5.E6 \\
\hline & $\Delta E_{\mathrm{c}}(\mathrm{eV})$ & 0,15 & 0,17 & \\
\hline \multirow{5}{*}{ Base-collecteur } & $J_{(}\left(\mathrm{A} / \mathrm{cm}^{2}\right)$ & 4. E-7 & 9. E-7 & 9.E-7 \\
\hline & $J_{\mathrm{r}}\left(\mathrm{A} / \mathrm{cm}^{2}\right)$ & 8.E-5 & 3. E-4 & 4. E-4 \\
\hline & $R_{\mathrm{s}}(\Omega)$ & 3,5 & 15 & 4 \\
\hline & $R_{\mathrm{p}}(\Omega)$ & $1,5 . \mathrm{E} 5$ & $3,5 . \mathrm{E} 4$ & 1.E5 \\
\hline & $\Delta E_{\mathrm{c}}(\mathrm{eV})$ & 0,11 & & \\
\hline \multirow{3}{*}{ Transistor } & $2 \mathrm{Ln}^{2} / \mathrm{W}_{\mathrm{b}}^{2}$ & & 135 & 135 \\
\hline & $\operatorname{Ln}(\mu \mathrm{m})$ & & 2,3 & 2,3 \\
\hline & $\tau \mathrm{n}(\mathrm{ns})$ & & 0,3 & 0,3 \\
\hline
\end{tabular}

$$
\begin{aligned}
& J=V^{*} / R_{\mathrm{P}}+J_{\mathrm{S}}(\left.\exp \left(V^{*} / n k_{\mathrm{B}} T\right)-1\right)+ \\
&+J_{\mathrm{R}}\left(\exp \left(V^{*} / 2 k_{\mathrm{B}} T\right)-1\right)
\end{aligned}
$$

Tableau I. - Paramètres utilisés pour ajuster les expressions théoriques aux résultats expérimentaux.

[Used parameters to fit the experimental results with the theoritical ones for the TBDH (no spacer), the TBHS (1 spacer) and the TBHDS (2 spacers). ] 
avec

$$
V^{*}=V-R_{\mathrm{S}} \cdot S \cdot J
$$

où $V$ est la tension appliquée et $S$ la surface de la jonction.

\subsubsection{Comparaison avec les résultats expérimen-} taux. - Nous avons ajusté les coefficients des expressions (9) et (10) afin de faire coïncider les valeurs calculées avec les valeurs mesurées. Deux exemples sont donnés sur la figure 3 et les valeurs des paramètres utilisés pour les trois transistors réalisés sont reportées dans le tableau $I$.

L'expression (2) permet de calculer la hauteur de la discontinuité de la bande de conduction qui est plus faible pour les jonctions enterrées (Tableau I). Le courant de recombinaison est quatre fois plus élevé pour la jonction enterrée. Ces résultats laissent supposer une moins bonne qualité pour la jonction enterrée ce qui peut s'expliquer par la mauvaise qualité des premières couches épitaxiées sur un matériau contenant de l'aluminium, ce composé jouant un rôle de getter pour les impuretés dues à l'environnement.

En prenant une mobilité de $7000 \mathrm{~cm}^{2} / \mathrm{V}$.s pour les électrons de la base d'épaisseur effective $W_{\mathrm{B}}=$ $0,28 \mu \mathrm{m}$ et avec $n_{i}=1,1 \times 10^{12} \mathrm{~cm}^{-3}$ l'expression (6) donne $J_{S}=1 \times 10^{-6} \mathrm{~A} / \mathrm{cm}^{2}$, valeur qui est en bon accord avec les valeurs utilisées pour les ajustements (Tableau I). Le courant de recombinaison est deux

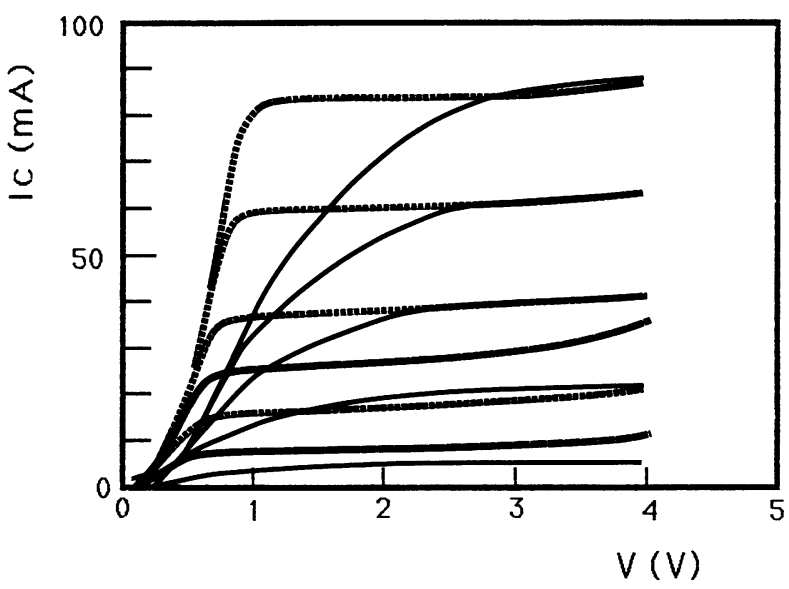

Fig. 5. - Caractéristiques expérimentales $I_{\mathrm{C}}\left(V_{\mathrm{CE}}\right)$ des transistors paramétrées en fonction du courant base. Traits pleins : transistor sans espaceur pour $I_{\mathrm{B}}$ égal à $1 ; 3$; $5 ; 7$ et $9 \mathrm{~mA}$. Pointillés : transistor à un espaceur pour $I_{\mathrm{B}}$ égal à 0,$5 ; 1 ; 1,5$ et $2 \mathrm{~mA}$. Tirets : transistor à deux espaceurs pour $I_{\mathrm{B}}$ égal à 0,2 et $0,4 \mathrm{~mA}$.

$\left[I_{\mathrm{C}}\left(V_{\mathrm{CE}}\right)\right.$ experimental characteristics for the transistors with the base current as a parameter. Full lines : transistor without spacer for $I_{\mathrm{B}}$ equal to $1 ; 3 ; 5 ; 7$ and $9 \mathrm{~mA}$. Dotted lines : transistor with one spacer for $I_{\mathrm{B}}$ equal to $0.5 ; 1 ; 1.5$ and $2 \mathrm{~mA}$. Dashed lines : transistor with two spacers for $I_{\mathrm{B}}$ equal to 0.2 and $0.4 \mathrm{~mA}$.] fois plus élevé pour les jonctions enterrées ce qui peut s'expliquer par les remarques précédentes.

Le courant de trou est négligeable dans les deux cas (terme pré-exponentiel de l'ordre de $10^{-12} \mathrm{~A} / \mathrm{cm}^{2}$ ).

Le courant de recombinaison est plus important pour les jonctions avec espaceur. Si on fait le raisonnement classique, c'est-à-dire que l'on suppose que la recombinaison se fait sur des centres situés dans la bande interdite suivant un processus de Shockley-Read, les centres les plus efficaces seront ceux qui sont situés en milieu de bande interdite et la recombinaison sera maximale lorsque le milieu de la bande interdite est à une énergie proche de celle du niveau de Fermi. Pour la jonction sans espaceur, cette coïncidence a lieu dans le semi-conducteur à grande bande interdite alors qu'elle a lieu dans le semi-conducteur à faible bande interdite pour la jonction avec espaceur. Le courant de recombinaison étant proportionnel à $n_{i}$, il sera donc supérieur dans l'hétérojonction avec espaceur. Néanmoins l'introduction d'un espaceur est intéressante du point de vue du coefficient d'injection, le courant de recombinaison n'étant que six fois plus élevé pour la jonction avec espaceur alors que le courant de saturation est vingt fois plus élevé.

\section{Caractérisation des transistors : phénomènes de collection.}

4.1 CARACTÉRISTIQUeS EXPÉRIMENTAles. - La figure 5 représente les caractéristiques $I_{\mathrm{C}}\left(V_{\mathrm{CE}}\right)$ des transistors réalisés. Nous remarquons un comportement très différent suivant que le collecteur comporte ou ne comporte pas d'espaceur. Dans ce dernier cas le courant de base est plus important (facteur 5 à 10) et le courant de collecteur se sature à une tension plus élevée. Pour essayer de comprendre un peu mieux les phénomènes de collection, nous avons dégagé ce qui, dans les résultats des mesures, correspond aux phénomènes parasites (résistances série et parallèle, courant de recombinaison). Ces calculs conduisent aux résultats reportés sur la figure 6 relatifs à un courant d'émetteur de $15 \mathrm{~mA}$. Pour les transistors avec un et deux espaceurs, le courant de recombinaison dans la base tend vers $0,11 \mathrm{~mA}$ ce qui conduit aux valeurs reportées dans le tableau I.

4.2 Résultats Des Simulations MonteCARLO. - Nous avons simulé avec un modèle unidimensionnel des transistors dont la géométrie correspond à celle des transistors réalisés (la discontinuité de la bande de conduction a été prise égale à $0,13 \mathrm{eV})$. Les jonctions émetteur-base avec et sans espaceur sont polarisées à des tensions conduisant à une densité de courant du même ordre de grandeur (respectivement 0,57 et $0,63 \mathrm{~V}$ ). Les jonctions base- 


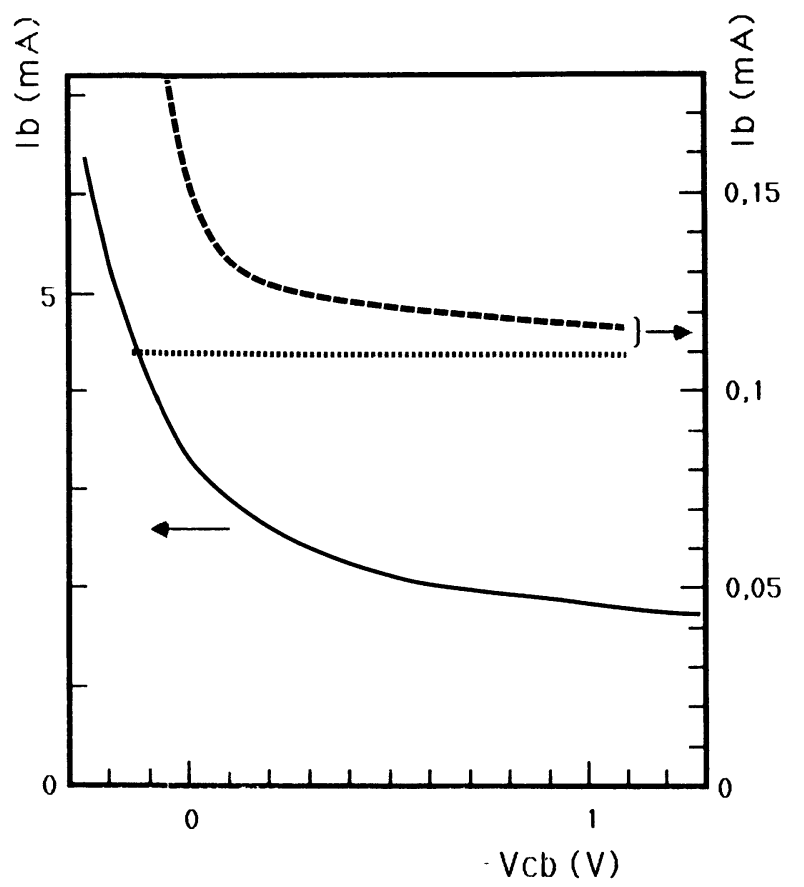

Fig. 6. - Courant de recombinaison dans la base en fonction de la tension collecteur-base. Traits pleins : transistor sans espaceur. Pointillés : transistor à un espaceur. Tirets : transistor à deux espaceurs.

[The recombination current in the base as a function of the collector-base bias voltage. Full line : transistor without spacer. Dotted line : transistor with a spacer. Dashed line : transistor with two spacers.]

collecteur sont polarisées à des tensions de $+0,05 \mathrm{~V}$ et $-0,45 \mathrm{~V}$.

Nous avons relevé tout au long des structures modélisées les densités d'électrons pour les deux polarisations base-collecteur. Les résultats sont reportés sur les figures 7,8 et 9 .

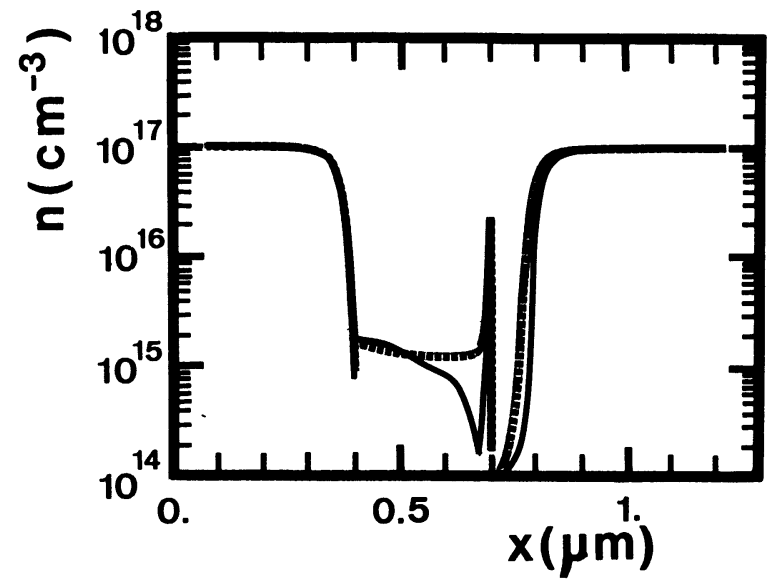

Fig. 7. - Densité des électrons tout au long du transistor sans espaceur. Traits pleins : $V_{\mathrm{BC}}$ égal à $0,05 \mathrm{~V}$. Pointillés : $V_{\mathrm{BC}}$ égal à $-0,45 \mathrm{~V}$.

[The electron density along the transistor without spacer. Full line : $V_{\mathrm{BC}}$ equal to $0.05 \mathrm{~V}$. Dotted line : $V_{\mathrm{BC}}$ equal to $-0.45 \mathrm{~V}$.

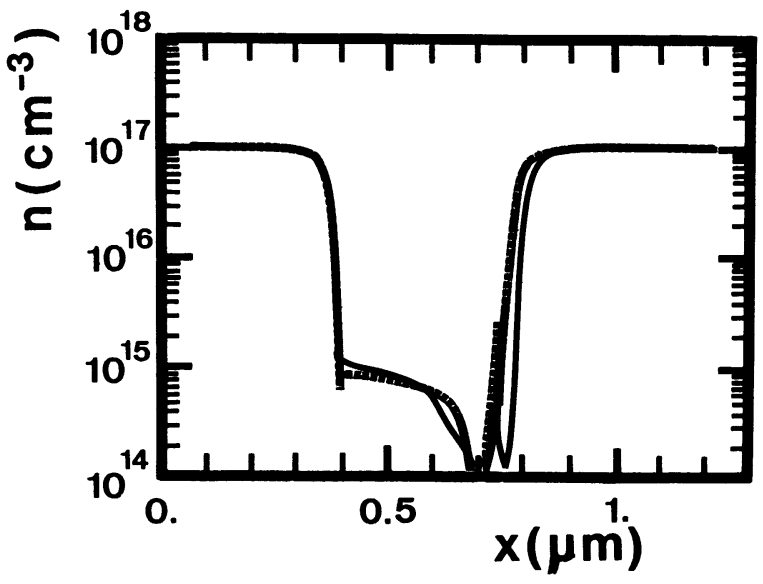

Fig. 8. - Densité des électrons tout au long du transistor à un espaceur. Traits pleins : $V_{\mathrm{BC}}$ égal à $0,05 \mathrm{~V}$. Pointillés : $V_{\mathrm{BC}}$ égal à $-0,45 \mathrm{~V}$.

[The electron density along the transistor with one spacer. Full line : $V_{\mathrm{BC}}$ equal to $0.05 \mathrm{~V}$. Dotted line : $V_{\mathrm{BC}}$ equal to $-0.45 \mathrm{~V}$.

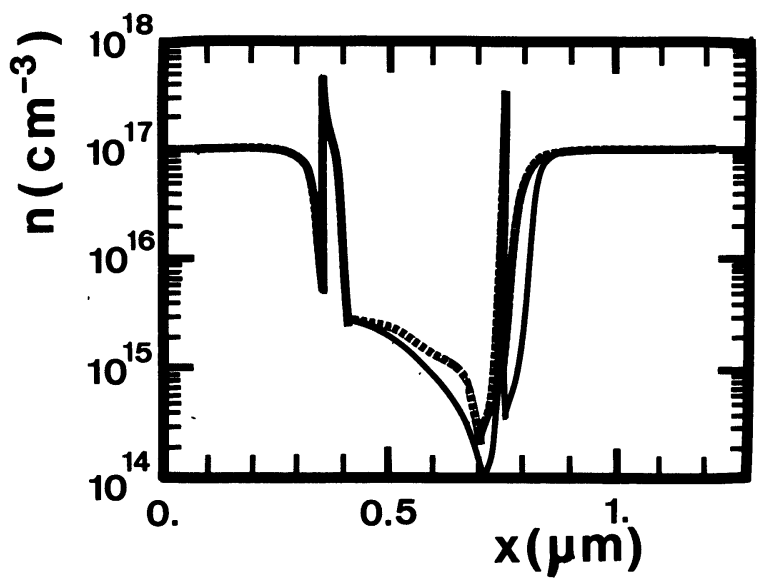

Fig. 9. - Densité des électrons tout au long du transistor à deux espaceurs. Traits pleins : $V_{\mathrm{BC}}$ égal à $0,05 \mathrm{~V}$. Pointillés : $V_{\mathrm{BC}}$ égal à $-0,45 \mathrm{~V}$.

[The electron density along the transistor with two spacers. Full line : $V_{\mathrm{BC}}$ equal to $0.05 \mathrm{~V}$. Dotted line : $V_{\text {BC }}$ equal to $-0.45 \mathrm{~V}$.]

Pour les transistors sans espaceur (Fig. 7) la densité d'électron dans la base diminue lorsque la tension base-collecteur croît, et ce particulièrement du côté du collecteur. Ce phénomène est dû à l'effacement progressif du spike (seuls les électrons ayant une énergie supérieure à celle du spike sont collectés : courant $3 /$ courant 4 , Fig. 1 ) et, si on suppose que le courant de recombinaison dans la base est proportionnel à la densité d'électron, explique la décroissance de $I_{\mathrm{B}}$ avec $V_{\mathrm{CB}}$ reportée sur la figure 6 .

Pour les transistors avec un espaceur au collecteur, la densité d'électron dans la base dépend très peu de la tension base-collecteur (Fig. 8). Dans ce cas les électrons, injectés par effet thermoionique à l'émetteur, sont chauds dans la base et sont parfaitement 
collectés malgré la présence d'une hétérojonction isotype au collecteur.

Pour les transistors avec deux espaceurs, la densité d'électrons diminue à mesure que la tension basecollecteur augmente (Fig. 9). Ce comportement peut s'expliquer par le fait que les électrons sont pratiquement thermiques dans la base et que leur collection est gênée par l'hétérojonction isotype du collecteur, cet effet étant de moins en moins important à mesure que $V_{\mathrm{CB}}$ augmente. Ce phénomène explique la décroissance de $I_{\mathrm{B}}$ avec $V_{\mathrm{CB}}$ (Fig. 6).

\section{Conclusions.}

Nous avons fabriqué et caractérisé deux types de Transistors Bipolaires à Hétérojonctions NnpnN InGaAlAs/InGaAs/InGaAlAs : des structures pour lesquelles les hétérojonctions sont abruptes et des structures pour lesquelles les hétérojonctions métallurgiques sont séparées des jonctions électriques par des espaceurs constitués d'une couche de semi-conducteur à faible bande interdite (InGaAs) de type $\mathrm{N}$ et nous avons analysé les résultats à l'aide de simulations Monte-Carlo. Ces espaceurs permettent de diminuer les hauteurs des barrières de potentiel vues par les électrons tout en conservant les hauteurs de barrière élevées vues par les trous ce qui permet d'accroître la part du courant participant à l'effet transistor par rapport au courant de recombinaison dans la zone de charge d'espace émetteurbase et ainsi d'obtenir des coefficients d'injection plus grands. L'étude des phénomènes d'injection nous a permis de montrer que celle-ci se faisait par effet thermoionique pour les hétérojonctions abruptes et par effet diffusif pour les hétérojonctions avec espaceur. D'autre part, la présence d'un espaceur favorise la collection, les transistors à hétérojonctions abruptes présentant un gain en courant en émetteur commun de l'ordre de 5, alors que ce gain atteint 100 pour les transistors comportant un espaceur au collecteur.

\section{Remerciements.}

Nous remercions G. Tremblay et D. Arquey qui ont réalisé les transistors et les membres de la division Microélectronique du laboratoire de Bagneux du CNET pour l'aide qu'ils nous ont apporté quant à la technologie des composés III-V et à la caractérisation des transistors. Nous sommes très reconnaissants à R. Castagné de l'Institut d'Electronique Fondamentale pour les nombreuses et fructueuses discussions que nous avons eues tout au long de cette étude. Ce travail est supporté par la DAII.

\section{Bibliographie}

[1] Kroemer, H., Proc. IEEE 17 (1982) 13.

[2] Malic, R. J., Hayes, J. R., CaPasso, F., Alavi, K., CHо, A. Y., IEEE Elect. Dev. Lett. 4 (1983) 383.

[3] Chaudhury, M., Tabatabai-Alavi, K., Fonstad, C. G., IEEE Elect. Dev. Lett. 5 (1984) 251.

[4] Su, L. M., Grote, N. G., Kaumanns, R., SchroeTER, H., Appl. Phys. Lett. 47 (1985) 28.

[5] Goldstein, L., Praseuth, J. P., Pelouard, J.-L., Hesto, P., 4th International Conference on Molecular Beam Epitaxy, York (1986).
[6] Dubon, C., Duchenois, A.-M., Bresse, J.-F., ANKRI, D., Elect. Lett. 21 (1985) 614.

[7] Ces algorithmes ont été développés à l'Institut d'Electronique Fondamentale (Université Paris-Sud). Voir par exemple : Hesto, P., Pône, J.-F., Mouis, M., Pelouard, J.-L., Castagné, R., Nasecode IV (Dublin), 1985, p. 315.

[8] Pelouard, J.-L., Hesto, P., Castagné, R., 10th IEEE/Cornell Conference (1985), p. 52. 